\title{
3
}

\section{Disfagia esofágica no idoso}

Eponina Maria de Oliveira Lemme

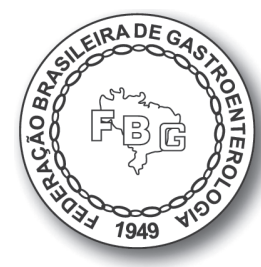

\section{INTRODUÇÃO}

A deglutição pode ser dividida em quatro fases:

- Preparatória oral: responsável pela ensalivação, mastigação e posicionamento do bolo alimentar na cavidade oral para o transporte para a faringe.

- Fase oral: voluntária, na qual ocorre a transferência do bolo até a faringe.

- Fase faríngea: responsável pelo transporte do bolo da faringe para o esôfago.

- Fase esofágica, em que ocorre o transporte do esôfago até o estômago.

Disfagia significa dificuldade de deglutição, mas o termo é utilizado para designar qualquer dificuldade entre o ato da deglutição até sua chegada ao estômago ${ }^{1}$.

Disfagia é um sintoma frequente na população geriátrica, sendo maior que na população em geral $(18 \% \text { vs. } 7 \%)^{2}$. Estima-se que 60 a $87 \%$ da população de residentes em casas de apoio tenham dificuldades na alimentação ${ }^{1}$. A disfagia nos idosos, ou seja, nos indivíduos de 60 anos ou mais, pode se tornar mais grave por problemas cognitivos, físicos e psiquiátricos, comuns nesta faixa etária, e piora o prognóstico de qualquer doença associada.

Alterações da fisiologia esofágica têm sido demonstradas em idosos, como anormalidades no esfíncter esofágico superior (EES) e faringe, aumento da frequência de falhas na peristalse esofágica, maior prevalência de hérnias hiatais e, consequentemente, baixa pressão do esfíncter esofágico inferior (EEI), além de alterações da percepção sensorial ${ }^{1,2}$. Entretanto, nem todos os idosos apresen- 
tam disfagia, fazendo crer que, embora existam alterações na idade avançada, elas nem sempre são sintomáticas.

Existem dois tipos básicos de disfagia em relação à sua localização e mecanismos fisiopatológicos: a disfagia orofaríngea (DOF) ou de transferência e a disfagia esofágica ou disfagia de transporte ${ }^{2,3}$. O idoso pode apresentar ambos os tipos de disfagia. Neste capítulo, será abordada a disfagia esofágica no idoso.

\section{DISFAGIA ESOFÁGICA OU DISFAGIA DE TRANSPORTE NO IDOSO}

$\mathrm{Na}$ disfagia esofágica ou de transporte, a dificuldade de passagem do alimento ocorre após o ato da deglutição ${ }^{3}$. As causas de disfagia esofágica no idoso podem ser de natureza orgânica, quando existe um distúrbio obstrutivo, seja de natureza intrínseca ou extrínseca, benigna ou maligna; ou de natureza funcional, quando a alteração responsável pelo sintoma é um distúrbio da motilidade esofágica. Estes distúrbios podem ser primários, quando a alteração motora esofágica é a própria manifestação da doença, ou secundários, se a doença de base é sistêmica e o comprometimento esofágico é apenas uma de suas manifestações ${ }^{4}$.

As causas mais frequentes de disfagia no idoso estão listadas no Quadro 1.

Quadro 1 Causas mais frequentes de disfagia esofagiana no idoso ${ }^{3}$

Alterações motoras

Acalásia

Espasmo difuso

Esclerodermia e outras associações reumatológicas

Dismotilidade ligada a refluxo

Motilidade ineficaz e outros distúrbios inespecíficos

Alterações estruturais

Carcinomas

Estenose péptica

Anéis

Compressão vascular

Estenose por medicamentos

\section{Características clínicas}

A anamnese é fundamental para o diagnóstico da disfagia esofágica, uma vez que, na maioria dos casos, o exame físico é pobre. Por ocorrer após o ato 
da deglutição, a disfagia esofágica não se acompanha de engasgos e é descrita na região retrosternal ou próxima ao apêndice xifoide. A disfagia, quando de natureza orgânica, é progressiva em relação à consistência alimentar, iniciando-se para sólidos, evoluindo para alimentos pastosos e finalmente líquidos.

Nas doenças funcionais, a disfagia também é de localização baixa, algumas vezes referida na região cervical (alta referida), mas sem engasgos. Ocorre tanto para alimentos sólidos como para alimentos líquidos e, dependendo da doença em questão, ela é intermitente ou lentamente progressiva. Fatores psíquicos como ansiedade e emoções podem agravar a disfagia.

\section{Diagnóstico}

O diagnóstico da disfagia esofágica no idoso, tal como na DOF, inicia-se pela suspeição, por meio de uma história cuidadosa ${ }^{2,3}$. As circunstâncias de início, duração e progressão da disfagia também fornecem importantes subsídios para o diagnóstico. O início súbito de disfagia para sólidos, acompanhado de sensação de desconforto torácico e sialorreia, sugere impactação de corpo estranho em áreas estenosadas benignas, de natureza péptica ou por esofagite medicamentosa, e ainda por anéis, sendo o mais frequente o anel de Shatzki, situado na junção esofagogástrica.

A estenose péptica no idoso pode ser a primeira manifestação da doença do refluxo gastresofágico (DRGE) neste grupo, uma vez que os sintomas típicos podem ser ocasionais ou passar despercebidos. A estenose por esofagite medicamentosa decorre do uso de vários medicamentos simultaneamente, inclusive os que são relacionados a esofagite química, como alendronato, quinidina, cloreto de potássio e antibióticos. A ingestão deste medicamentos em posição supina e com pouco líquido, os estreitamentos naturais do esôfago que se tornam mais presentes no idoso e a xerostomia são os responsáveis por esta complicação.

$\mathrm{Na}$ maioria das vezes, entretanto, o paciente apresenta-se cronicamente doente. Disfagia progressiva com importante emagrecimento em curto espaço de tempo, antecedentes de tabagismo e etilismo sugerem lesão maligna.

Disfagia exclusiva para sólidos de longa duração ou lentamente progressiva é característica das estenoses benignas. Muitas vezes, o paciente adapta-se a outras consistências alimentares e mantém o estado geral. Antecedentes de pirose e regurgitação ácida apontam para estenose péptica; histórico associado de alterações cutâneas, articulares e/ou fenômeno de Raynaud sugere colagenoses.

Disfagia intermitente para sólidos e líquidos, entremeada de episódios de dor torácica, sugere alteração funcional, por exemplo espasmo esofágico difuso, que pode acometer a faixa etária acima dos 60 anos $^{5}$. 
Disfagia para sólidos e líquidos de longa duração, com estado geral do paciente relativamente conservado, acompanhada de regurgitações de material salivar ou alimentar, surgindo até horas após as refeições, sugere acalasia. As regurgitações não raramente surgem à noite, provocando tosse, engasgos, sensação de sufocação e, eventualmente, infecção respiratória. Idosos com acalasia podem se desnutrir com o passar do tempo, não sendo incomum que se apresentem até com grau de caquexia crônica, permanecendo restritos ao leito, com possibilidade de aspiração decorrente das regurgitações. Nas fases iniciais da doença, pode haver dor torácica espontânea que melhora com ingestão de líquidos, podendo preceder o surgimento da disfagia ${ }^{5}$. Um estudo comparativo demonstrou que idosos com acalasia têm tempo de doença maior e menos pirose e dor torácica do que os não idosos ${ }^{6}$.

O exame físico de idosos com disfagia esofágica pode mostrar evidências de doenças sistêmicas associadas, possíveis sinais metastáticos, em casos de lesão maligna e até desnutrição, na dependência da intensidade e duração do quadro. Os pacientes com disfagia funcional mantêm o estado geral, em sua maioria.

\section{Métodos complementares}

A investigação complementar da disfagia esofágica deve se iniciar com a endoscopia digestiva alta (EDA), em casos de suspeita de disfagia orgânica ${ }^{3}$. As neoplasias são observadas e biopsiadas, sendo fundamental o diagnóstico histopatológico na condução da terapêutica. Os anéis e as estenoses podem ser percebidos e, como se verá adiante, a endoscopia tem também importante papel na terapêutica.

Os diferentes graus de esofagite podem ser avaliados; o esôfago de Barrett apresenta mucosa característica que permite coleta de material para diagnóstico definitivo, assim como para verificar a presença e o tamanho de uma hérnia hiatal. Não raramente, idosos apresentam volumosas hérnias que podem ser a causa de disfagia pelo seu tamanho, inclusive as paraesofágicas.

O estudo radiológico (esofagografia ou videoesofagografia) é fundamental nas disfagias crônicas, principalmente nas de origem funcional, pois permite demonstrar alterações anatômicas e estruturais, avaliar o tempo de trânsito, a presença de contrações anormais e o calibre do esôfago ${ }^{2,3}$. Na acalasia, é possível avaliar os diferentes graus de megaesôfago, sendo classificados de I a IV, conforme o diâmetro do esôfago ${ }^{6}$. Diâmetro de anéis, extensão de estenoses e hérnias hiatais volumosas são mais bem avaliados com o estudo radiológico.

A esofagomanometria (EMN) é o método de escolha para o diagnóstico definitivo dos distúrbios motores do esôfago $(\mathrm{DME})^{4,5}$. Sua maior aplicação é na confirmação do diagnóstico de acalasia. Os outros DME apresentam padrão 
característico já consagrado na literatura internacional ${ }^{4}$. A maior parte dos DME pode ter associação com DRGE, devendo ser avaliada a necessidade de prosseguir a investigação com o emprego de pHmetria esofágica prolongada, caso não haja esofagite erosiva importante.

Mais recentemente, a manometria de alta resolução (MAR), empregando sonda dotada de inúmeros sensores de pressão (32-36) posicionados muito próximos um do outro, tem fornecido resultados interessantes na abordagem dos DME, com possibilidade de diagnóstico de alterações que não podem ser percebidas pela manometria convencional. A mais importante delas é a obstrução funcional da junção esofagogástrica (OFJEG) que obedece a causas múltiplas, entre elas, a disfagia pós-fundoplicatura, podendo ser exclusivamente funcional. Seu diagnóstico é feito quando a pressão integrada de relaxamento (IRP, sigla em inglês), uma medida obtida com o método, está elevada ( $\geq 15$ $\mathrm{mmHg}$ ), refletindo uma anormalidade do relaxamento da junção. A peristalse esofágica está preservada na maioria dos pacientes. Alguns acreditam que a OFJEG possa representar uma síndrome de acalasia incompleta ${ }^{7}$. Outra contribuição importante da MAR é a divisão de acalasia em três grupos (I, II e III) de acordo com o grau de pressurização. O tipo I é a acalasia clássica, sem pressurização; o II é a acalasia com pressurização; o tipo III é a acalasia espástica ${ }^{8}$.

\section{TRATAMENTO}

- Disfagia orgânica: as causas mais frequentes de disfagia orgânica nos idosos são o carcinoma epidermoide do esôfago e a estenose péptica, complicação da $\mathrm{DRGE}^{3}$. No primeiro caso, após estadiamento adequado, o tratamento envolve radioterapia, cirurgia e possivelmente quimioterapia coadjuvante. A estenose de natureza péptica é manejada com dilatações instrumentais, notadamente com as velas de Savary, de calibres progressivos. O número de dilatações é variável e depende do calibre e da extensão da estenose, sendo o objetivo do procedimento a melhora da disfagia ${ }^{9}$. É importante o tratamento antirrefluxo intensivo, com inibidor de bomba de prótons e medidas comportamentais e posturais concomitantes ao procedimento de dilatação. Estenoses por pílulas ou de outras causas também são manejadas com dilatações.

- Disfagia funcional: em torno de $20 \%$ dos portadores de acalasia têm mais de 60 anos $^{3,6} . \mathrm{Na}$ avaliação de um idoso com acalasia, é fundamental excluir doença orgânica, como a evidência de um carcinoma de fundo gástrico invadindo o esôfago. A dilatação pneumática da cárdia (DPC) é o procedimento de escolha para tratamento definitivo da acalasia no idoso ${ }^{3}$. É um 
procedimento ambulatorial que emprega, de modo geral, um balão de baixa complacência (Rigiflex), posicionado por via endoscópica após sedação consciente. As técnicas de DPC variam de acordo com a instituição. Em nosso serviço, são empregados os balões de 3, 3,5 e $4 \mathrm{~cm}$, que podem ser utilizados em uma mesma sessão ou em sessões diferentes, dependendo da laceração produzida pela insuflação e da melhora da disfagia ${ }^{10}$. Bons resultados da DPC são encontrados em 60 a $80 \%$ no curto e no médio prazos e são melhores em indivíduos acima de 40 anos do que nos mais jovens ${ }^{11}$. $\mathrm{O}$ tratamento cirúrgico por miotomia laparoscópica com fundoplicatura parcial é outra opção de tratamento, menos comumente empregada em idosos, podendo ser utilizada em caso de falha da DPC.

Estudos empregando a MAR têm mostrado melhor resposta a qualquer forma de tratamento na acalasia tipo II, pior resposta na tipo III e intermediária na tipo $\mathrm{I}^{8}$. Acredita-se que estas contribuições da MAR na acalasia devam ser confirmadas por nossos pesquisadores, em virtude da enorme experiência que o país tem neste assunto. Um estudo em andamento em nosso serviço não mostrou diferenças nos resultados precoces do tratamento de acalasias I e II com DPC. Não se conhecem estudos com MAR exclusivamente em idosos.

Mais recentemente, um novo método vem sendo oferecido para o tratamento da acalasia. Trata-se da miotomia endoscópica peroral, mais conhecida por sua sigla em inglês POEM. A POEM consiste em atingir a camada muscular circular do esôfago por meio de um túnel criado com auxílio do endoscópio, após uma pequena abertura feita na mucosa, complementada com miotomia próximo à junção esofagogástrica ${ }^{12}$. A técnica é sofisticada, demanda curva de aprendizado e deve ser realizada apenas por profissionais experientes. Trabalhos recentes têm demonstrado 80 a $90 \%$ de bons resultados no curto caso, comparáveis aos do tratamento cirúrgico ${ }^{13,14}$, porém referem taxa de refluxo por pHmetria entre 30 e $50 \%{ }^{13,14}$. Não há referência ao emprego da POEM exclusivamente em idosos.

Drogas que reduzem a pressão do EEI, como a nifedipina e os nitratos, também podem ser usadas com cuidado em idosos com acalasia, por via sunlingual (SL), 15 a 30 minutos antes das refeições, enquanto não se programa o tratamento definitivo. Os efeitos colaterais, como cefaleia e hipotensão, frequentemente limitam seu emprego ${ }^{3}$.

Pacientes com comorbidades, elevado risco cirúrgico ou muito desnutridos podem ser submetidos ao tratamento com aplicação de toxina botulínica (BoTox) no EEI ${ }^{15}$. Esta droga bloqueia a liberação de acetilcolina na junção neuromuscular, reduzindo a pressão do EEI e melhorando a disfagia. Tem sido empregada como ponte para o tratamento definitivo, pois, muitas vezes, o grau 
de desnutrição do paciente torna real o risco de morte em caso de complicação de um procedimento terapêutico. $\mathrm{O}$ inconveniente da BoTox é o seu curto prazo de ação, devendo ser repetida a curtos intervalos ${ }^{15}$.

Entre os DME não acalasia, para a OFJEG tem sido proposta conduta expectante, evitando métodos invasivos, uma vez que, em muitos pacientes, há uma resolução espontânea da disfagia ${ }^{16}$. Entretanto, têm sido descritos tratamentos com aplicação de BoTox, DPC e até miotomia de Heller com fundoplicatura para os casos resistentes ${ }^{7}$. O espasmo esofágico difuso, intitulado de espasmo esofágico distal nos dias atuais à $\mathrm{MAR}^{17}$, pode ser encontrado em idosos. $\mathrm{O}$ tratamento clínico deste distúrbio motor envolve também a administração de nitratos, antagonistas de canal de cálcio ou sildenafil para alívio da disfagia e/ ou da dor torácica ${ }^{17}$. Como os sintomas são intermitentes, o uso de medicação contínua não isenta de efeitos colaterais pode não ser desejável. Cuidados gerais para manuseio de disfagia, como comer devagar, mastigar bem e consumir pequenas porções, devem ser enfatizados e muitos obtêm melhora com esta conduta. Injeção endoscópica de BoTox no músculo esofágico também é uma opção interessante em casos selecionados de espasmo distal que não melhoram com tratamento clínico ${ }^{17}$.

Finalmente, é importante assinalar que pacientes com espasmo distal e também os que apresentam achados manométricos de motilidade ineficaz e esfíncter hipotenso ${ }^{4}$ podem ter DRGE associada, não raramente com grandes hérnias hiatais, e o tratamento específico pode se acompanhar de melhora da disfagia. Exclusão de refluxo ou o seu tratamento, caso presente, são muito importantes na condução terapêutica de idosos com disfagia esofágica.

\section{CONSIDERAÇÕES FINAIS}

A disfagia esofágica no idoso pode ter causas orgânicas e funcionais. Entre as primeiras, destacam-se as neoplasias, seguidas de estenoses benignas, sendo a da DRGE complicada a mais comum. Nas causas funcionais, acalasia pode ser encontrada em idosos, assim como alterações motoras ligadas a DRGE. O diagnóstico inicia-se com a suspeição por uma história cuidadosa, seguida de investigação complementar pertinente, que orienta o tratamento adequado.

\section{REFERÊNCIAS BIBLIOGRÁFICAS}

1. Cook IJ. Oropharyngeal dysphagia. Gastroenterol Clin North Am. 2009;38:411-31.

2. Aslam M, Vaezi MF. Dysphagia in the elderly. Gastroenterol \& Hepatology. 2013;9:784-95.

3. Achem SR, DeVault KR. Dysphagia in aging. J Clin Gastroenterol. 2005;39(5):357-71.

4. Spechler SJ, Castell DO. Classification of oesophageal motility abnormalities. Gut. 2001;49:145-51. 
5. Domingues GRS, Lemme EMO. Manifestações clínicas dos distúrbios motores esofagianos. Rev Bras Med. 2000;57:239-45.

6. Schechter R, Lemme EM, Novais P, Biccas B. Achalasia in the elderly people: a comparative study. Arq Gastroenterol. 2011;48:19-23.

7. Scherer JR, Kwiatek MA, Soper NJ, Pandolfino JE, Kahrilas PJ. Functional esophagogastric junction obstruction with intact peristalsis: a heterogeneous syndrome sometimes akin to achalasia. J Gastrointest Surg. 2009;13(12):2219-25.

8. Kahrilas PJ, Bredenoord AJ, Fox M, Gyawali CP, Roman S, Smout AJ, et al. The Chicago classification of esophageal motility disorders v 3.0. Neurogastroenterol Motil. 2015;27(2):160-74.

9. Novais P, Lemme EMO, Equi C, Medeiros C, Lopes C, Vargas C. Estenoses benignas do esôfago: tratamento com dilatador de Savary-Gilliard. Arq Gastroenterol. 2008;45:290-4.

10. Borges AA, Lemme EM, Abrahao LJ Jr., Madureira D, Andrade MS, Soldan M, et al. Pneumatic dilation of the cardia or laparoscopic esophageal myotomy for the treatment of achalasia: factors related to a good response. Dis Esophagus. 2014;27(1):18-23.

11. Campos GM, Vittinghoff E, Rabl CA, Takata M, Gadenstätter M, Lin F et al. Endoscopic and surgical treatments for achalasia: a systematic review and meta-analysis. Ann Surgery. 2009;249(1):4557.

12. Inoue H, Minami H, Kobayashi Y, Sato Y, Kaga M, Suzuki M, et al. Peroral endoscopic myotomy (POEM) for esophageal achalasia. Endoscopy. 2010;42(4):265-71.

13. Sharata AM, Dunst CM, Pescarus R, Shlomovitz E, Wille AJ, Reavis KM, et al. Peroral endoscopic myotomy (POEM) for esophageal primary motility disorders: analysis of 100 consecutive patients. J Gastrointestinal Surg. 2015;19(1):161-70.

14. Sanaka MR, Hayat U, Thota PN, Jegadeesan R, Ray M, Gabbard SL, et al. Efficacy of peroral endoscopic myotomy vs other acalasia treatments in improving esophageal function. World J Gastroenterol. 2016;22(20):4918-25.

15. Pasricha PJ, Rai R, Ravich WJ. Botulinum toxin for achalasia: long term outcome and predictors of response. Gastroenterology. 1996;110:1410-5.

16. Pérez-Fernandez MT, Santander C, Marinero A, Burgos-Santamaría D, Chavarría-Herbozo C. Characterization and follow-up of esophagogastric junction outflow obstruction detected by high resolution manometry. Neurogastroenterol Motil. 2016;28(1):116-26.

17. Roman S, Kahrilas PJ. Distal esophageal spasm. Dysphagia. 2012;27:114-23. 